\title{
ENERGY RELAXATION OF HOT ELECTRONS AND INELASTIC COLLISION TIME IN THIN METAL FILMS AT LOW TEMPERATURES
}

\author{
S.I. Dorozhkin*, F. Lell and W. Schoepe \\ Institut fur Angewandte Physik, Universitat Regensburg D-8400 Regensburg, West Germany \\ (Received 23 April 1986 by M. Cardona)

\begin{abstract}
In a sandwich consisting of a thin $\mathrm{Au}$ film and a thin Bi film insulated by a $500 \AA \mathrm{SiO}$ layer the electrons of one film are heated above the lattice temperature while in the other film they are kept in thermal equilibrium with the phonons. By measuring the magnetoresistance of both films we obtain the temperature difference between electrons and phonons from which we imply the energy-relaxation time $\tau_{\epsilon}$ from $0.3 \mathrm{~K}$ to $2 \mathrm{~K}$. We find that electron-phonon scattering determines $\tau_{\epsilon}$ and, above $5 \mathrm{~K}$, also the inelastic collision time $\tau_{i}$ obtained from weak localization theory in thermal equilibrium.
\end{abstract}

WEAK LOCALIZATION PHENOMENA in thin metal films have revealed detailed information about the dynamics of the electron gas because they are sensitive to various scattering mechanisms which break the phase coherence of the electrons [1]. Especially from the magnetoresistance (MR) these scattering times can easily be determined. A particularly simple situation exists in the limit of strong (or negligible) spin-orbit scattering where the MR is almost exclusively governed by inelastic collisions of the electrons [2]. Therefore, the $\mathrm{MR}$ in thin $\mathrm{Au}$ or $\mathrm{Bi}$ films, e.g., provides a direct measurement of the inelastic scattering time $\tau_{i}$ [3-7]. From the temperature dependence of $\tau_{i}$ one usually concludes that at high temperatures $(T \gtrsim 2 \mathrm{~K}) \tau_{i}$ is caused by electron-phonon collisions. At lower temperatures, however, $\tau_{i}$ is thought to be given by inelastic electron-electron scattering. This now offers the interesting possibility of heating the electron gas to a temperature $T_{e}$ above the low phonon temperature $T_{p}$. While $T_{e}$ is established on a time scale of the order of $\tau_{i}$ the energy relaxation is slower because it requires collisions between electrons and phonons to cool the electron gas. Overheating of the electrons therefore is possible up to a temperature where electron-phonon collisions become as frequent as electron-electron collisions. If both temperatures $T_{e}$ and $T_{p}$ can be measured simultaneously one can determine the energy-relaxation time $\tau_{\epsilon}$ which has not been accessible under equilibrium conditions where $\tau_{i}\left(\ll \tau_{\epsilon}\right)$ sets the limit for phase coherence.

As a thermometer for the electron gas we use the $\mathrm{MR}$ of thin $\mathrm{Au}$ and $\mathrm{Bi}$ films. Both films are thermally

*Permanent address: Institute of Solid State Physics, Academy of Sciences of the USSR, Chernogolovka, Moscow district, USSR. tightly coupled by a $500 \AA$ insulating SiO layer so that they have a common phonon bath because the phonon wavelength in our temperature regime $(0.3$ to $2 \mathrm{~K})$ is larger than $500 \AA[8]$. Driving one film at successively higher d.c. current levels results in a reduction of its MR because of an increase of $T_{e}$. Simultaneously we measure the MR of the other film at a sufficiently low current level to ensure equilibrium between electrons and phonons and obtain the common phonon temperature $T_{p}$. From $T_{e}-T_{p}$ we can infer the energy relaxation rate of the electrons which has a temperature dependence indicative of electron-phonon scattering. Furthermore we find that $\tau_{\epsilon}$ extrapolates to the high temperature $\tau_{i}$ curve, consistent with dominant electron-phonon scattering above $5 \mathrm{~K}$. Thus, our method provides a first direct comparison of $\tau_{\epsilon}$ and $\tau_{i}$ in thin metal films.

Our sample is fabricated by first depositing a $75 \AA$ $\mathrm{Au}$ film onto a glass substrate. Except for the two current and three voltage connections this film is covered by the SiO layer on top of which we deposit a $200 \AA \mathrm{Bi}$ film. The length between the voltage probes is $3 \mathrm{~mm}$ and the width of the films is $1 \mathrm{~mm}$. Typical convenient resistivities are about $300 \Omega / \square$ for either film. Homogeneity is checked by interchanging voltage connections and comparing resistivities in different sections of the films. Insulation between the films is usually better than $20 \mathrm{M} \Omega$. The sample is mounted inside the ${ }^{3} \mathrm{He}$ pot of our cryostat and is completely immersed in the liquid in order to keep the lattice temperature as low as possible. A transverse magnetic field up to 3.5 tesla can be applied for MR measurements, which are performed with a fourterminal a.c. bridge at low frequencies $(32 \mathrm{~Hz})$ and low current levels $(\sim 5 \mu \mathrm{A})$. Heating of the electron gas of one film is achieved by applying a d.c. current $I$ of up to $1 \mathrm{~mA}$ and measuring the voltages with a high resolution 


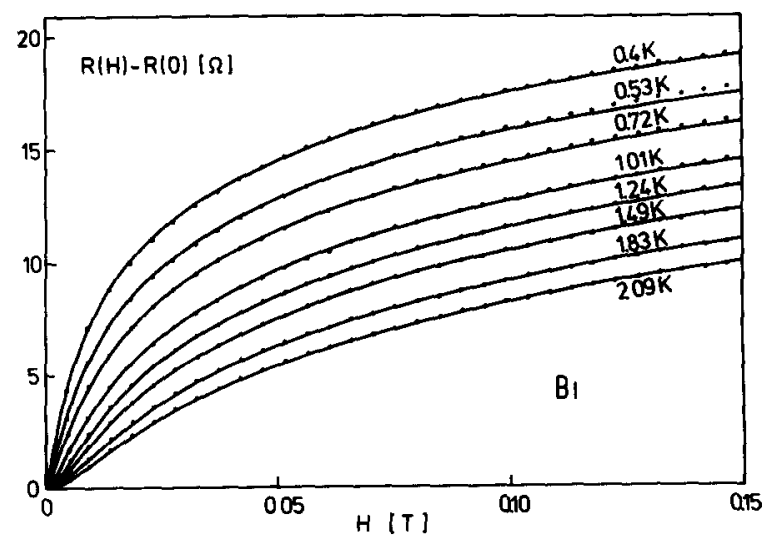

Fig. 1. Magnetoresistance of the $\mathrm{Bi}$ film at different temperatures. Solid lines are the measurements, dots are from a fit of weak localization theory.

d.c. voltmeter while the other film is being monitored at low a.c. levels as before. The power $I^{2} R$ delivered to the electron gas heats up $T_{e}$ and to a lesser extent also $T_{p}$ and ultimately the ${ }^{3} \mathrm{He}$ bath.

We first measure the equilibrium MR of both films at various temperatures between 0.3 and $20 \mathrm{~K}$. These data not only provide us with the temperature calibration of the MR but in addition enable us to evaluate the inelastic scattering time $\tau_{i}(T)$ by fitting the standard theory of weak localization ot the data. Fig. 1 shows a typical set of MR data at low fields where the MR is determined by weak localization only (at higher fields effects from electron-electron interaction may contribute). In the limit of strong spin-orbit scattering the MR is described simply by [2]

$\frac{R(H)-R(0)}{R(0)}=\frac{\mathrm{e}^{2}}{2 \pi^{2} \hbar} \cdot R_{\square} \cdot \frac{1}{2} f\left(\frac{H}{H_{i}}\right)$,

where $f(x)=\psi(1 / 2+1 / x)+\ln x$ and $\psi$ is the digamma function. The "inelastic field" $H_{i}$ is related to $\tau_{i}$ by

$H_{i} \tau_{i}=\hbar / 4 \mathrm{e} D$,

with the diffusion coefficient $D$ being given by the Einstein relation $D=\left(\mathrm{e}^{2} R_{\square} d N\left(\epsilon_{F}\right)\right)^{-1} ; d$ is the film thickness and $N\left(\epsilon_{F}\right)$ is the density-of-states at the Fermi level. Equation (1) may be extended to allow for finite spin-orbit scattering times [2]. These corrections, however, are not essential here. From a fit to the data we obtain $H_{i}$ from which we calculate $\tau_{i}$ by using published values for the density-of-states $N\left(\epsilon_{F}\right)$ [9]. The results are depicted in Figs. 2 and 3.

Application of a large d.c. current then heats the electrons in one film to a temperature $T_{e}$ above the phonon temperature $T_{p}$. Both temperatures can now be determined from the temperature calibration of the MR as measured before. For small temperature differences

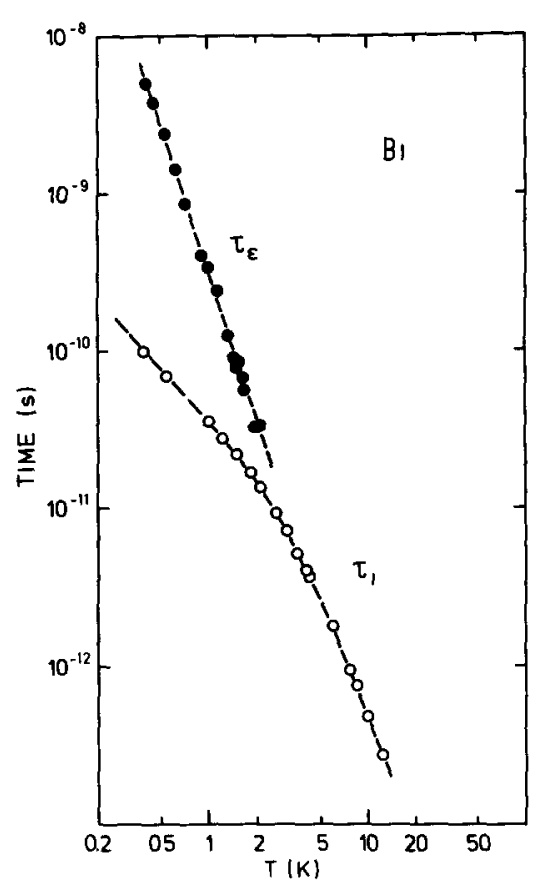

Fig. 2. Energy-relaxation time $\tau_{\epsilon}$ and inelastic collision time $\tau_{i}$ in bismuth.

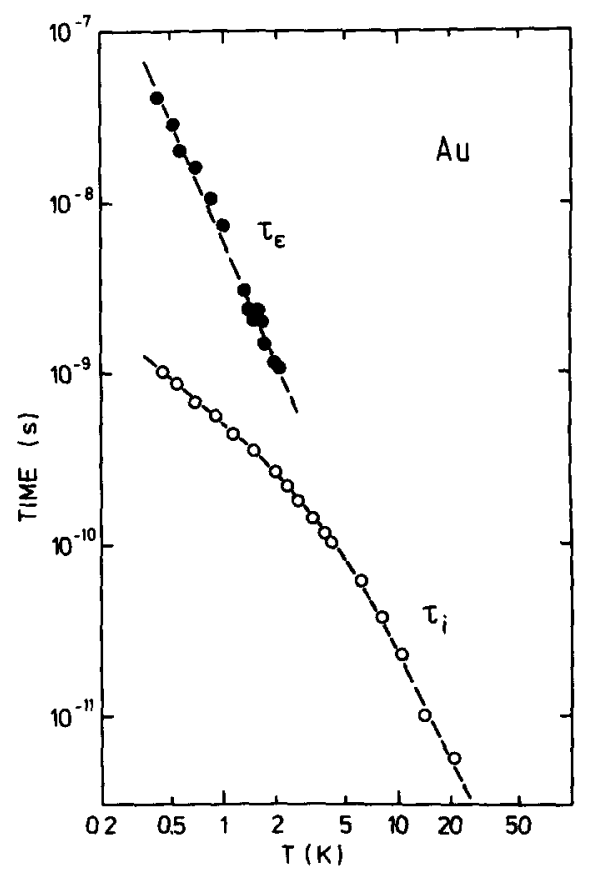

Fig. 3. Energy-relaxation time $\tau_{\epsilon}$ and inelastic collision time $\tau_{i}$ in gold.

$\Delta T=T_{e}-T_{p}$ we calculate $\tau_{\epsilon}$ from a simple heating model [10]:

$I^{2} R \tau_{\epsilon}(T)=V_{0} C_{\mathrm{el}}(T) \Delta T$, 
where $V_{0}$ is the volume of the film and $C_{\mathrm{el}}(T)=\gamma_{\mathrm{el}} T$ is the electronic specific heat at the average temperature $T=\left(T_{e}+T_{p}\right) / 2$. It is interesting to note that the densityof-states is a common scaling factor for both $\tau_{i}$ and $\tau_{\epsilon}$ (via $\gamma_{\mathrm{el}}$ ) and does not affect their ratio. By varying the current from $10 \mu \mathrm{A}$ to $1 \mathrm{~mA}$ (i.e. four orders of magnitude in power) we obtain the data shown in Figs. 2 and 3.

Analyzing our results we find that in both metals $\tau_{i} \propto T^{-p}$ has two distinct regimes: $p=1.0$ below $2 \mathrm{~K}$ for both $\mathrm{Au}$ and $\mathrm{Bi}$, and above $5 \mathrm{~K} p=2.0$ for $\mathrm{Au}$ and $p=2.5$ for $\mathrm{Bi}$, although the temperature intervals outside of the transition regime are rather short for a precise determination of the power laws. We attribute the low temperature behavior of $\tau_{i}$ to inelastic electronelectron scattering, where in fact a $T^{-1}$ law has been calculated $[11,12]$. Our measured values at $1 \mathrm{~K}$ are a factor of 4 smaller for $\mathrm{Bi}$ and a factor of 2 larger for Au when compared with [12]. The high temperature part reflects electron-phonon processes for which the predicted power laws depend on the purity and dimensionality of the sample [13]. With regard to phonons we consider our sample to be three-dimensional because of the coupling to the substrate. The purity is given by $q_{p} \cdot l_{0}$, where $q_{p}$ is a typical phonon wavevector and $l_{0}$ is the elastic mean free path. Estimating $l_{0}$ from the diffusion coefficient and the Fermi velocity and setting $q_{p} \sim k_{B} T / \hbar c$, where $c$ is the velocity of sound, we find that our Au film is "dirty" at all of our temperatures $\left(q_{p} l_{0} \ll 1\right)$, whereas our Bi film may be "clean" above $0.5 \mathrm{~K}\left(q_{p} l_{0} \gtrsim 1\right)$. For the clean case a $T^{-3}$ law is expected $[14,15]$. For the dirty case, however, the situation is unclear since both a $T^{-2}$ law or a $T^{-4}$ law have been predicted $[1,13,14]$.

Comparing our $\tau_{i}$ data with the earlier experiments [3-5] on $\mathrm{Bi}$, we note good agreement in temperature dependence as well as absolute values. As for the Au film the published work $[1,6,7]$ gives a less clear picture. Obviously the investigated samples are difficult to compare. This might be related to the more granular structure of the Au film.

The essential result of our work is the determination of the energy relaxation time $\tau_{\epsilon}$. In Bi we find $\tau_{\epsilon} \propto T^{-3.0}$, which gives clear evidence of electron-phonon scattering. In $\mathrm{Au}$ we get $\tau_{\epsilon} \propto T^{-2.2}$ which may be consistent with the dirty limit. Furthermore, it is most remarkable that in both films $\tau_{\epsilon}$ clearly coincides with a low temperature extrapolation of the $\tau_{i}$ data from above $5 \mathrm{~K}$. This proves that electron-phonon scattering limits phase coherence at high temperatures. The absolute values of $\tau_{\epsilon}$ at $1 \mathrm{~K}$ are $3.4 \cdot 10^{-10} \mathrm{~s}$ in $\mathrm{Bi}$ and $7.2 \cdot 10^{-9} \mathrm{~s}$ in $\mathrm{Au}$, which is of the expected order when compared with theory [15] or with measurements of electron-phonon scattering rates by resonance methods at higher temperatures [16].
Earlier experiments on electron heating in thin films $[17,7]$ have been analyzed by use of equation (2) under the assumption $T_{e} \gg T_{p}$ and by replacing the equilibrium temperature $T$ by $T_{e}$ in the $\ln T$-divergence of the zero field resistance. From a comparison of the measured voltage dependence of the resistance with the equilibrium temperature dependence on can imply $\tau_{\epsilon}$ to scale as $T^{-p^{\prime}}$ with $p^{\prime}=3$ in $\mathrm{Au}-\mathrm{Pd}$ films [18] and $p^{\prime} \simeq 0.4$ 0.8 in Au films [7] . Obviously, our method of separately measuring $T_{e}$ and $T_{p}$ does not rely on the above assumption.

By employing a completely different technique Roukes et al. [19] recently measured $\tau_{\epsilon} \propto T^{-3}$ in thick $\mathrm{Cu}$ films at very low temperatures. $T_{e}$ and $T_{p}$ were determined by noise thermometry on two separate films on the same substrate. In that work $\tau_{i}$ was not measurable.

In summary, our method yields a direct comparison of $\tau_{\epsilon}$ and $\tau_{i}$ by use of weak localization. Electron electron scattering governs $\tau_{i}$ at low temperatures and hence limits the phase coherence time, whereas electronphonon scattering determines $\tau_{\epsilon}$ and, at higher temperatures, also $\tau_{i}$.

Acknowledgements - It is a pleasure to thank K.F. Renk for continuing support and encouragement. One of us (S.I.D.) gratefully acknowledges a grant from the Alexander von Humboldt Foundation.

\section{REFERENCES}

1. For a review see, e.g., G. Bergmann, Phys. Rep. 107, 1 (1984), and references cited therein.

2. S. Hikami, A.I. Larkin \& Y. Nagaoka, Prog. Theor. Phys. 63, 707 (1980).

3. F. Komori, S. Kobayashi \& W. Sasaki, J. Phys. Soc. Japan. 52, 368 (1983).

4. A.K. Savchenko, A.S. Rylik \& V.N. Lutskii, $Z h$. Eksp. Teor. Fiz. 85, 2210 (1983) [Sov. Phys. JETP 58, 1281 (1983)].

5. E.I. Bukhshtab, A.V. Butenko, Yu.F. Komnik \& V.V. Pilipenko, Solid State Commun. 53, 347 (1985).

6. T. Kawaguti \& Y. Fujimori, J. Phys. Soc. Japan. 52, 722 (1983).

7. S.I. Dorozhkin \& V.T. Dolgopolov, Pis'ma $Z h$. Eksp. Teor. Fiz. 36, 15 (1982) [Sov. Phys. JETP Lett. 36, 18 (1982)].

8. Estimating the phonon wavelength $\lambda_{B} \sim h c / k_{B} T$ at $1 \mathrm{~K}$ a sound velocity $c>10^{3} \mathrm{~m} \mathrm{~s}^{-1}$ gives $\lambda_{p}>$ $500 \AA$.

9. For $N\left(\epsilon_{F}\right)$ we take $1.14 \cdot 10^{47} \mathrm{~J}^{-1} \mathrm{~m}^{-3}$ for $\mathrm{Au}$ and $1.57 \cdot 10^{45} \mathrm{~J}^{-1} \mathrm{~m}^{-3}$ for $\mathrm{Bi}$, see N.E. Phillips, Phys. Rev. 118, 664 (1960).

10. P.W. Anderson, E. Abrahams \& T.V. Ramakrishnan, Phys. Rev. Lett. 43, 719 (1979).

11. E. Abrahams, P.W. Anderson, P.A. Lee \& T.V. Ramakrishnan, Phys. Rev. B24, 6783 (1981).

12. B.L. Altshuler, A.G. Aronov \& D.E. Khmel'nitskii, J. Phys. C15, 7367 (1982). 
13. See, e.g., D.E. Prober, Percolation, Localization, and Superconductivity, (Edited by A.M. Goldman and S.A. Wolf) Plenum Press, New York (1984).

14. For a review see, e.g., A. Schmid, Localization, Interaction, and Transport Phenomena, (Edited by B. Kramer, G. Bergmann, and Y. Bruynseraede) Springer-Verlag, Berlin (1985).

15. W.E. Lawrence \& A.B. Meador, Phys. Rev. B18 1154 (1978).

16. V.F. Gantmakher, Rep. Prog. Phys. 37, 317
(1974).

17. G.J. Dolan \& D.D. Osheroff, Phys. Rev. Lett. 43, 721 (1979).

18. G.J. Dolan, Inhomogeneous Superconductors, (Edited by D.D. Gubser, T.L. Francavilla, S.A. Wolf, and J.R. Leibowitz) American Institute of Physics, New York (1980).

19. M.L. Roukes, M.R. Freeman, R.S. Germain, R.C. Richardson \& M.B. Ketchen, Phys. Rev. Lett. 55, 422 (1985). 